\title{
InCoB2014: mining biological data from genomics for transforming industry and health
}

\author{
Christian Schönbach ${ }^{1,2}$, Tin Wee $\operatorname{Tan}^{3}$, Shoba Ranganathan ${ }^{4^{*}}$ \\ From Asia Pacific Bioinformatics Network (APBioNet) Thirteenth International Conference on Bioinformatics \\ (InCoB2014) \\ Sydney, Australia. 31 July - 2 August 2014
}

\begin{abstract}
The 13th International Conference on Bioinformatics (InCoB2014) was held for the first time in Australia, at Sydney, July 31-2 August, 2014. InCoB is the annual scientific gathering of the Asia-Pacific Bioinformatics Network (APBioNet), hosted since 2002 in the Asia-Pacific region. Of 106 full papers submitted to the BMC track of InCoB2014, 50 (47.2\%) were accepted in BMC Bioinformatics, BMC Genomics and BMC Systems Biology supplements, with three papers in a new BMC Medical Genomics supplement. While the majority of presenters and authors were from Asia and Australia, the increasing number of US and European conference attendees augurs well for the international flavour of InCoB. Next year's InCoB will be held jointly with the Genome Informatics Workshop (GIM), September 9-11, 2015 in Tokyo, Japan, with a view to integrate bioinformatics communities in the region.
\end{abstract}

\section{Introduction}

The 13th InCoB (International Conference on Bioinformatics), an official conference of the Asia-Pacific Bioinformatics Network (APBioNet) [1], was held in Sydney, Australia. In keeping with InCoB's tradition to locate the conference in different Asia-Pacific countries, InCoB went to Australia, for the first time, with the NSW Chief Scientist and Engineer, Professor Mary O'Kane giving the opening address. The conference was well attended by the local community as well as participants from Asia-Pacific, Europe and USA, with scientists from Qatar and Turkey attending InCoB for the first time. The plenary talks covered mutations in DNA, proteomics, RNA Biology, systems biology, mathematics, statistics and computer science as well as bioinformatics training to equip scientists with the current "tools of the trade" and best practice methodologies, while the conference attendees presented cutting edge research with three industry presentations, 89 orals as well as 45 posters.

\footnotetext{
* Correspondence: shoba.ranganathan@mq.edu.au

${ }^{4}$ Department of Chemistry and Biomolecular Sciences and ARC Centre of Excellence in Bioinformatics, Macquarie University, Sydney NSW 2109,

Australia

Full list of author information is available at the end of the article
}

\section{Manuscript submission and review}

We offered authors four tracks to submit manuscripts for potential publication in the supplement issues of BMC Bioinformatics, BMC Systems Biology or BMC Genomics (BMC track) and PeerJ [2]. Of the 112 submitted manuscripts, 106 were in the BMC track. All manuscripts received at least two reviews from the 92 member Program Committee, supported by 35 additional reviewers (Additional file 1). The first round of reviews resulted in the provisional acceptance of 12 (11.3\%) manuscripts, with minor revisions. The authors of 45 manuscripts, including three that were transferred by the Program Committee Co-chairs to the PeerJ track, had to address major concerns raised by the reviewers. After a second round of review, another 38 (35.8\%) manuscripts were provisionally accepted pending minor revisions. In all cases, the reviewers assessed the manuscripts at least twice and all submissions were ranked based on the reviewers' scores, in accord with earlier InCoB publications [3].

The 20 articles in this supplement cover mainly "genomic" topics, with three medically-oriented papers going into BMC Medical Genomics [4] for the first time. The InCoB2014 BMC Bioinformatics supplement [5] comprises 16 manuscripts while another 11 papers are presented in the BMC Systems Biology supplement [6]. 
Five more submissions have been published in the InCoB2014 PeerJ collection [7].

\section{Sequencing, genomes and genome analysis}

With genome sequencing technologies becoming more and more accessible and affordable, the genetic origin of the Marwari horse was established by whole genome sequencing [8] while the chloroplast genome of Australia's macadamia nut tree [9]. funRNA [10] is a collection of RNA interference (RNAi) genes, implicated in genome defence as well as diverse cellular, developmental, and physiological processes, from fungal, metazoan and plant genomes along with bacterial and archaeal genomes. Abbas et al. [11] have assessed de novo assembly software for fungal genome data. Proteogenomics is increasingly used for the accurate annotation of protein coding regions using proteomic data. As currently available proteogenomic tools are tailored specifically for human and eukaryotic data, Uszkoreit et al. [12] have developed a proteogenomic analysis pipeline, specifically for bacterial genomes.

\section{Transcriptomics}

Seven papers are devoted to transcriptome analysis addressing a range of challenges from epigenetics $[13,14]$ to understanding transcript-level changes with diseases [15-18]. dCAP [13] is a new method to simultaneously detect constitutive and differential regulation of multiple epigenetic factors from multiple sample datasets, while the YNA database [14] provides an integrated data mining platform for chromatin changes in yeast. Huang et al. [15] have identified transcript-level changes implicated in biological dysfunction of energy metabolism and hemostasis in schizophrenia while Yarmishyn et al. [16] have pinpointed a non-coding RNA as a novel marker of neuroblastoma progression. Sheng et al. [17] have identified the most common microRNA (miRNA) editing events in colon cancer. For non-small cell lung cancer, Mah et al. [18] have identified a single single nucleotide polymorphism (SNP) as a good prognostic marker of patient outcome following chemotherapy. While transcripts are in the main used as a measure of protein expression, Sun $e t$ al. [19] have applied transcriptomics and pathway analysis to improve protein identification from proteomics data, especially where the sample size is limited to a few cells.

\section{Functional genomics}

Guanine-rich nucleotide sequences form four-stranded Gquadruplex structures. Yano and Kato [20] have used hidden Markov Models to reliably identify these structures, especially those involved in DNA transcription. Wu et al. [21] have developed a novel algorithm (GM-SMCC), for predicting the functional annotation of protein-coding genes, applied successfully to the 2001 KDD cup yeast gene datasets. Small ubiquitin-like modifier (or SUMO) proteins covalently attached other proteins lead to sumoylation, which is involved in various cellular processes, including nuclear-cytosolic transport, transcriptional regulation, apoptosis, protein stability, response to stress, and cell cycle progression. Yavuz and Sezerman [22] have developed an accurate support vector machine (SVM)based approach to identify these sites from sequence data, as a precursor to experimental validation.

\section{Pharmacogenomics}

In the era of genomic medicine, it is possible that some approved drug molecules can be used for diseases other than those they were originally approved for. Yang and coworkers [23] propose the concept of "Homopharma", to combine similar drug binding environments to better understand molecular binding mechanisms for deploying approved drugs for other diseases, known as "repurposing." Tyagi et al. [24] have optimized arylthioindole compounds for efficiently disrupting tubulin assembly towards anti-cancer therapy, using QSAR and molecular dynamics approaches.

\section{Disease informatics}

Taguchi and co-workers [25] have identified TINAGL1 and B3GALNT1 as novel key candidate genes in the treatment of non-small cell lung cancer from gene expression and epigenetic data, while sets of microRNA biomarkers for detecting lung squamous cell carcinoma have been proposed by Song et al. [26]. Xu et al. [27] have developed a novel methodology, MHC2MIL for developing peptidebased vaccines, benchmarked on 12 HLA DP and DQ molecules.

\section{Medical informatics}

Papers specifically relating on genome-scale analysis with a disease focus are presented in a new supplement in BMC Medical Genomics and a brief overview of these articles is presented here. With pandemic viral infections spreading rapidly by jet travel, understanding cross-species transmissibility of vectors is addressed by Tan and co-workers [28] for influenza A, using a random forest approach. In the quest for diagnostic biomarkers and therapeutic targets, Nenadic and co-workers [29] have developed a novel text-mining approach to successfully identify novel genes and pathways for thyroid cancer subtypes. While gene-based cancer biomarkers are typically sets of hundreds or thousands of genes, Olsen et al. [30] have analysed public proteogenomic data to zoom in on just 32 tumor antigenic proteins as biomarkers for invasive ductal carcinomas. These studies point to the combined use of several "-omic" technologies as the focus of future studies for understanding, detecting and combatting diseases. 


\section{Conclusion}

With the growth in regional bioinformatics meetings, including ISCB-Asia meetings and the 2013 IEEE International Conference on Bioinformatics and Biomedicine (BIBM) in China [31], there is a growing community of bioinformatics scientists in the Asia-Pacific. To consolidate multiple meetings and to provide cross-talk between traditionally different bioinformatics communities, we invite you to attend the $2015 \mathrm{InCoB}$ meeting to be held jointly with the Genome Informatics Workshop (GIW) in Tokyo, Japan [32].

\section{Additional material}

Additional file 1: List of Program Committee Members and Additional Reviewers in Alphabetical Order.

\section{Competing interests}

The authors declare that they have no competing interests.

\section{Authors' contributions}

SR wrote the introduction. CS and SR (Program Committee Co-chairs) managed the review and editorial processes, respectively. TWT supported the post-acceptance manuscript processing.

\section{Acknowledgements}

We are indebted to all members of the Program Committee and additional reviewers for their efforts and time. We are grateful to the NSW T\&l Conference grant awarded to SR for hosting InCoB2014, our sponsors: the Australian Bioinformatics Network, the International Society for Computational Biology, Qiagen, Millennium Science and ABSciEx, and for material support from Macquarie University and Sydney Business Events. Special thanks go to ASN Events for running the conference smoothly. Finally, we are deeply grateful to Isobel Peters and Jennifer Egar of BMC who have supported us through the supplement publication process.

This article has been published as part of BMC Genomics Volume 15 Supplement 9, 2014: Thirteenth International Conference on Bioinformatics (InCoB2014): Computational Biology. The full contents of the supplement are available online at http://www.biomedcentral.com/bmcgenomics/ supplements/15/S9.

\section{Authors' details}

${ }^{1}$ Department of Biology, School of Science and Technology, Nazarbayev University, Astana 010000, Republic of Kazakhstan. ${ }^{2}$ Center for AIDS Research, Kumamoto University, Kumamoto 860-0811, Japan. ${ }^{3}$ Department of Biochemistry, Yong Loo Lin School of Medicine, National University of Singapore, Singapore 117599. ${ }^{4}$ Department of Chemistry and Biomolecular Sciences and ARC Centre of Excellence in Bioinformatics, Macquarie University, Sydney NSW 2109, Australia.

Published: 8 December 2014

\section{References}

1. The Asia-Pacific Bioinformatics Network. [http://www.apbionet.org].

2. PeerJ. [http://peerj.com].

3. Schönbach C, Shen B, Tan T, Ranganathan S: InCoB2013 introduces Systems Biology as a major conference theme. BMC Syst Biol 2013, 7(Suppl 3):S1.

4. Thirteenth International Conference on Bioinformatics (InCoB2014): Medical Genomics [http://www.biomedcentral.com/bmcmedgenomics/supplements/ 7/S3].

5. Thirteenth International Conference on Bioinformatics (InCoB2014): Bioinformatics [http://www.biomedcentral.com/bmcbioinformatics/ supplements/15/S16].
6. Thirteenth International Conference on Bioinformatics (InCoB2014): Systems Biology [http://www.biomedcentral.com/bmcsystbiol/supplements/8/S4].

7. InCoB 2014 Collection - hosted on PeerJ. [https://peerj.com/collections/ 10-incob2014/].

8. Jun J, Cho YS, Hu H, Kim HM, Jho S, Gadhvi P, Park KM, Lim J, Paek WK, Han K, Manica A, Edwards JS, Bhak J: Whole genome sequence and analysis of the Marwari horse breed and its genetic origin. $B M C$ Genomics 2014, 15(Suppl 9):S4.

9. Nock CJ, Baten AKM, King GJ: Complete chloroplast genome of Macadamia integrifolia confirms the position of the Gondwanan earlydiverging eudicot family Proteaceae. BMC Genomics 2014, 15(Suppl 9): S13.

10. Choi J, Kim KT, Jeon J, Wu J, Song $H$, Asiegbu FO, Lee $Y H$ : funRNA: a fungicentered genomics platform for genes encoding key components of RNAi. BMC Genomics 2014, 15(Suppl 9):S14.

11. Abbas MM, Malluhi QM, Balakrishnan P: Assessment of de novo assemblers for draft genomes: a case study with fungal genomes. BMC Genomics 2014, 15(Suppl 9):S10.

12. Uszkoreit J, Plohnke N, Rexroth S, Marcus K, Eisenacher M: The bacterial proteogenomic pipeline. BMC Genomics 2014, 15(Suppl 9):S19.

13. Chen $K B$, Hardison $R$, Zhang $Y$ : dCaP: detecting differential binding events in multiple conditions and proteins. BMC Genomics 2014, 15(Suppl 9):S12.

14. Hung PC, Yang TH, Liaw HJ, Wu WS: The Yeast Nucleosome Atlas (YNA) database: an integrative gene mining platform for studying chromatin structure and its regulation in yeast. BMC Genomics 2014, 15(Suppl 9):S5.

15. Huang KC, Yang KC, Lin H, Tsao TTH, Lee SA: Transcriptome alterations of mitochondrial and coagulation function in schizophrenia by cortical sequencing analysis. BMC Genomics 2014, 15(Suppl 9):S6.

16. Yarmishyn AA, Batagov AO, Tan JZ, Sundaram GM, Sampath $P$, Kuznetsov VA, Kurochkin IV: HOXD-AS1 is a novel IncRNA encoded in HOXD cluster and a marker of neuroblastoma progression revealed via integrative analysis of noncoding transcriptome. BMC Genomics 2014, 15(Suppl 9):S7.

17. Zheng Y, Li T, Ren R, Shi D, Wang S: Revealing editing and SNPs of microRNAs in colon tissues by analyzing high-throughput sequencing profiles of small RNAs. BMC Genomics 2014, 15(Suppl 9):S11.

18. Mah TZ, Yap XNA, Limviphuvadh V, Li N, Srinath S, Kuralmani V, Feng M, Liem N, Adhikari S, Yong WP, Soo RA, Maurer-Stroh S, Eisenhaber F, Tong JC: Novel SNP improves differential survivability and mortality in non-small cell lung cancer patients. BMC Genomics 2014, 15(Suppl 9):S20.

19. Sun J, Zhang GL, Li S, Ivanov AR, Fenyo D, Lisacek F, Murthy SK, Karger BL, Brusic V: Pathway analysis and transcriptomics improve protein identification by shotgun proteomics from samples comprising small number of cells - a benchmarking study. BMC Genomics 2014, 15(Suppl 9):S1.

20. Yano M, Kato Y: Using hidden Markov models to investigate G-quadruplex motifs in genomic sequences. BMC Genomics 2014, 15(Suppl 9):S15.

21. Wu Q, Ye Y, Ho SS, Zhou S: Semi-supervised multi-label collective classification ensemble for functional genomics. BMC Genomics 2014, 15(Suppl 9):S17.

22. Yavuz AS, Sezerman OU: Predicting sumoylation sites using support vector machines based on various sequence features, conformational flexibility and disorder. BMC Genomics 2014, 15(Suppl 9):S18.

23. Chiu YY, Tseng JH, Liu KH, Lin CT, Hsu KC, Yang JM: Homopharma: a new concept for exploring the molecular binding mechanisms and drug repurposing. BMC Genomics 2014, 15(Suppl 9):S8.

24. Tyagi C, Gupta A, Goyal S, Dhanjal JK, Grover A: Fragment based group QSAR and molecular dynamics mechanistic studies on arylthioindole derivatives targeting the $\alpha-\beta$ interfacial site of human tubulin. $B M C$ Genomics 2014, 15(Suppl 9):S3.

25. Umeyama H, Iwadate M, Taguchi YH: TINAGL1 and B3GALNT1 are potential therapy target genes to suppress metastasis in non-small cell lung cancer. BMC Genomics 2014, 15(Suppl 9):S2.

26. Song R, Liu Q, Hutvagner G, Nguyen H, Ramamohanarao K, Wong L, Li J: Rule discovery and distance separation to detect reliable miRNA biomarkers for the diagnosis of lung squamous cell carcinoma. BMC Genomics 2014, 15(Suppl 9):S16.

27. Xu Y, Luo C, Qian M, Huang X, Zhu S: MHC2MIL: a novel multiple instance learning based method for MHC-II peptide binding prediction by considering peptide flanking region and residue positions. BMC Genomics 2014, 15(Suppl 9):S9. 
28. Eng CLP, Tong JC, Tan TW: Predicting host tropism of influenza A virus proteins using random forest. BMC Medical Genomics 2014, 7(Supp 3):S1

29. Wu C, Schwartz JM, Brabant G, Nenadic G: Molecular profiling of thyroid cancer subtypes using large-scale text mining. BMC Medical Genomics 2014, 7(Supp 3):S3.

30. Olsen LR, Campos B, Winther O, Sgroi DC, Karger BL, Brusic V: Tumor antigens as proteogenomic biomarkers in invasive ductal carcinomas. BMC Medical Genomics 2014, 7(Supp 3):S2.

31. BIBM. 2013 [http://bibm2013.tongji.edu.cn/]

32. GIW-InCoB. 2015 [http://incob.apbionet.org/incob15].

doi:10.1186/1471-2164-15-S9-11

Cite this article as: Schönbach et al:: InCoB2014: mining biological data from genomics for transforming industry and health. BMC Genomics 2014 15(Suppl 9):11.

Submit your next manuscript to BioMed Central and take full advantage of:

- Convenient online submission

- Thorough peer review

- No space constraints or color figure charges

- Immediate publication on acceptance

- Inclusion in PubMed, CAS, Scopus and Google Scholar

- Research which is freely available for redistribution

Submit your manuscript at www.biomedcentral.com/submit 